Additional Perspectives articles for Influenza: The Cutting Edge book collection are available at http://perspectivesinmedicine.cshlp.org/cgi/collection/influenza_the_cutting_edge.

\title{
Bat-Borne Influenza A Viruses: An Awakening
}

\author{
Kevin Ciminski ${ }^{1,2}$ and Martin Schwemmle ${ }^{1,2}$ \\ ${ }^{1}$ Institute of Virology, Medical Center University of Freiburg, 79104 Freiburg, Germany \\ ${ }^{2}$ Faculty of Medicine, University of Freiburg, 79104 Freiburg, Germany \\ Correspondence: martin.schwemmle@uniklinik-freiburg.de
}

Influenza A viruses (IAVs) originating from aquatic waterfowl recurrently cross interspecies barriers, which is greatly facilitated by utilizing cell surface-exposed monosaccharide sialic acids located on vertebrate cells as a universal host cell receptor. These glycan structures are first bound by the viral hemagglutinin (HA) for cell entry and then cleaved by the viral neuraminidase (NA) for particle release. In contrast, viruses of the recently identified batborne IAV subtypes $\mathrm{H} 17 \mathrm{~N} 10$ and $\mathrm{H} 18 \mathrm{~N} 11$ encode $\mathrm{HA}$ and NA homologs unable to interact with sialic acid residues despite a high degree of structural homology with their conventional counterparts. However, the most recent findings show that bat IAV HAs make use of the major histocompatibility complex class II proteins of different vertebrate species to gain entry into host cells, potentially permitting a broader host tropism. This review recapitulates current progress in the field of bat IAV research including the first assessment of the spillover potential of these bat viruses into other mammals.

$\mathrm{R}^{\mathrm{c}}$ ecurrent transmissions to various host species associated with a remarkably fast host adaptation are undoubtedly an outstanding characteristic of influenza A viruses (IAVs) (Johnson et al. 2017). A long-existing paradigm poses that all known IAV subtypes originate from aquatic waterfowl from which they cross the interspecies barrier to circulate in mammalian and avian species (Webster et al. 1992). Ironically, bats, which harbor a higher proportion of dangerous and life-threatening zoonotic viruses than any other mammalian order (Calisher et al. 2006; Hayman 2016; Olival et al. 2017), were long unrecognized and disregarded as a potential host for influenza viruses. The view of aquatic waterfowl as the sole host of all known IAV subtypes was challenged when in
2012 three complete genomes, belonging to a same but yet undescribed influenza virus strain, were identified on pan-influenza screening approaches in rectal swap samples of the frugivorous bat species Sturnira lilium from Guatemala (Tong et al. 2012). Genome comparisons and phylogenetic analysis revealed that all genomic segments, with the exception of the sequence coding for the surface glycoprotein neuraminidase (NA), are most closely related to those of classical IAVs (Tong et al. 2012). Because of the extensive divergence in NA and the fact that the isolated hemagglutinin (HA) sequence did not match any known IAV HA, the virus was then provisionally classified as H17N10 subtype. In the subsequent year, another previously unknown influenza genome was found in the feces

Editors: Gabriele Neumann and Yoshihiro Kawaoka

Additional Perspectives on Influenza: The Cutting Edge available at www.perspectivesinmedicine.org

Copyright (C) 2021 Cold Spring Harbor Laboratory Press; all rights reserved; doi: 10.1101/cshperspect.a038612

Cite this article as Cold Spring Harb Perspect Med 2021;11:a038612 
of the Peruvian flat-faced fruit bat Artibeus planirostris (Tong et al. 2013). Strikingly, this novel bat-derived influenza genome was phylogenetically closely related to the H17N10 genome, but the HA and NA encoding sequences showed again an extraordinary divergence from classical $\mathrm{HA}$ and NA sequences, wherefore they were provisionally designated as $\mathrm{H} 18$ and N11, respectively (Tong et al. 2013). To date, two additional genomic sequences of the H18N11 subtype have been detected in the Brazilian bat species Artibeus lituratus (Campos et al. 2019), indicating perpetual circulation of bat IAVs. Consistently, seroepidemiological data indicated a high prevalence of $\mathrm{H} 17$ (38\%) and H18 HAspecific antibodies (50\%) in various screened Central and South American bats, showing that infections with these viruses are not limited to individual bat species (Tong et al. 2013). Interestingly, despite comprehensive surveys, no bat influenza genomes have yet been detected in European bats (Fereidouni et al. 2015). Whether this hints toward a restricted circulation among bats in the Americas remains to be determined. Phylogenomic analyses were performed to backdate the chronological events that led to the emergence of these bat IAVs. This study indicated that classic IAVs (subtypes H1-16 and N1-9) and bat-derived IAVs (subtypes H17-18 and N10-11) share a common precursor from which both lineages probably separated at the end of the sixteenth century and underwent independent evolution from then on (Tong et al. 2013; Worobey et al. 2014). However, it remains elusive whether New World bats harbor ancient forms of influenza viruses that were preserved in this exclusive environment, or whether these viruses evolved to fit the specific demands of their hosts (Brunotte et al. 2016). Meanwhile, bats in Ghana were found to be positive for antibodies against the H9 subtype (Freidl et al. 2015), and in 2019 a classical IAV strain of the H9N2 subtype was isolated from the Egyptian fruit bat Rousettus aegyptiacus (Kandeil et al. 2019). As this recently reported H9N2 subtype resembles a classical IAV in terms of its receptor specificity and phylogenetic relationship, it will not be discussed in detail here. Rather, we will focus on the two divergent H17N10 and H18N11 subtypes, hereinafter designated as bat IAVs. In the following, we will review recent findings supporting the concept that despite their independent evolution, bat and classical IAVs have maintained the same fundamental structure in terms of virion architecture, genomic organization, and their encoded proteins. Nevertheless, bat IAVs are characterized by several remarkable features that are unprecedented among all known classical influenza virus strains.

\section{CONVENTIONAL AND BAT IAVs, A PRIME EXAMPLE OF CONVERGENT EVOLUTION}

The genomes of bat and classical IAVs are similarly composed of eight single-stranded RNAs (viral RNAs [vRNAs]) in antisense orientation, each of them encapsidated by multiple copies of the viral nucleoprotein (NP) and terminally bound by the heterotrimeric RNA-dependent RNA polymerase (RdRP) forming a viral ribonucleoprotein (vRNP) complex (Compans et al. 1972; Palese and Schulman 1976; Hsu et al. 1987; Fodor et al. 1993; Tong et al. 2012, 2013). All vRNAs encompass a central monoor polycistronic protein-coding region, which is flanked at the $3^{\prime}$ and $5^{\prime}$ terminal ends by a short noncoding regions (NCRs). These NCRs together with parts of the respective vRNA coding region constitute the packaging sequences that build a specific RNA-RNA interaction network among the vRNPs to presumably drive incorporation of eight individual viral genome segments into progeny virions (Fujii et al. 2003; Watanabe et al. 2003; Hutchinson et al. 2010; Goto et al. 2013). On co-infection of host cells with different parental viruses, the segmented nature of the virus' genome allows the exchange of gene segments to generate genetically distinct descendants. This process, known as genetic reassortment, is a main feature of IAV and can give rise to numerous novel genotypes, thereby facilitating rapid adaptation to new host environments. However, reassortment requires both an RNAand protein-based compatibility between the parental viruses to ensure, first, sufficient genome packaging and, subsequently, viral fitness (White and Lowen 2018). 
Initial studies that evaluated the zoonotic and reassortment potential of the H17N10 and H18N11 subtypes were performed in 2014 by Juozapaitis and colleagues and Zhou and colleagues, who independently generated chimeric bat viruses by reverse genetics (Juozapaitis et al. 2014; Zhou et al. 2014). These chimeras comprised the six so-called internal gene segments (PB2, PB1, PA, NP, M, and NS) of either H17N10 or H18N11 together with segments encoding the HA and NA glycoprotein sequences of the conventional IAV strains A/Puerto Rico/8/1934 (H1N1) (PR8), A/swine/Texas/ 4199-2/1998 (H3N2), or A/SC35M (H7N7). Generation of these infectious chimeric viruses required the respective bat IAV $\mathrm{HA}$ and NA packaging sequences at the terminal ends of the classical HA and NA coding region. Using this approach, both groups showed that genomic reassortment between bat and conventional IAV gene segments does not occur readily, because of an underlying genome-packaging incompatibility at the nucleotide level. Importantly, this block in gene reassortment is not limited to the HA and NA genome segment but was also observed for all other segments with the exception of the $M$ segment (Zhou et al. 2014; Yang et al. 2017). Whether this observation reflects the highly conserved nature of the $\mathrm{M}$ segment and its gene products and/or the partial compatibility of the packaging signals between bat and conventional IAV remains unclear. In contrast, the gene segments of the two divergent bat subtypes H17N10 and H18N11 were highly interchangeable and formed various reassortants (Zhou et al. 2014).

The failure of bat chimeric viruses to reassort with conventional IAV is not exclusively a consequence of RNA incompatibility, but also due to a mismatch of the internal viral proteins. Although the bat PB2, PB1, and PA polymerase proteins share a relatively high degree of amino acid identity with their counterparts of classical human or avian IAVs (Tong et al. 2012, 2013; Pflug et al. 2014), their functional compatibility with conventional IAV polymerase proteins is limited. While the PB2 of bat IAV supports the activity of H1N1, H5N1, H5N9, and H7N7 polymerases, PB1 and PA do so only in rare indi- vidual cases (Juozapaitis et al. 2014; Poole et al. 2014; Zhou et al. 2014). In contrast, bat IAV NP is more promiscuous as it was functionally complementarily in various IAV polymerases of human and avian origin (Juozapaitis et al. 2014; Zhou et al. 2014). Notably, the exchange of influenza B virus polymerase proteins or NP with those of bat IAV did not result in functional RdRP complexes (Zhou et al. 2014). Aside from the bat IAV polymerase proteins, only individual exchanges with the matrix protein 1 and the nuclear export protein (NEP) were tolerated in the context of classical IAV (Juozapaitis et al. 2014), and interestingly an infectious recombinant PR8 virus encoding the NS1 but not the NEP gene of H17N10 could be generated (Zhao et al. 2016).

Further studies on the bat H17N10 NP in the context of a classical H7N7 virus unveiled an unexpected but fundamental complexity of influenza virus reassortment and genome packaging. Although bat IAV NP fully supported the function of classical IAV polymerases of various subtypes including H7N7, recombinant H7N7 viruses encoding H17N10 NP were not viable, despite flanking the bat IAV NP coding region with authentic H7N7 packaging sequences (Moreira et al. 2016b). Site-specific exchange of as little as seven H7N7 NP amino acids located in the head domain with the corresponding ones found in H17N10 NP ultimately allowed the generation of a viable $\mathrm{H} 7 \mathrm{~N} 7$ virus encoding a mutant NP segment. These amino acid mutations in NP caused a severe attenuation and selectively altered genome packaging of four of the eight vRNPs (Moreira et al. 2016b). Strikingly, further mutational analysis showed that different genomic subsets were incorrectly packaged when H17N10 amino acids were introduced into the H7N7 NP body domain. Therefore, although the precise molecular mechanism in mediating the interplay of NP amino acids and vRNA is currently unknown, this study showed for the first time that both packaging sequences and specific amino acids in NP act in concert to guarantee a coordinated packaging process. It furthermore suggests that circulation of bat and conventional IAVs in different, nonoverlapping host species might have driven the selection 
K. Ciminski and M. Schwemmle

of different sets of matching pairs of packaging sequences and NP amino acids (Ciminski et al. 2017).

\section{BAT IAV HA PROTEINS EVOLVED TO USE MHC CLASS II MOLECULES}

Influenza virus HAs are the most abundant viral surface glycoprotein and are responsible for host cell attachment and subsequent cell entry. On the viral surface, HA is present as a noncovalently linked homotrimer, consisting of a proximal stalk region and a distal globular head domain that mediates binding to either $\alpha 2,3$ or $\alpha 2,6$-linked sialic acid residues (Fig. 1) (Sauter et al. 1989; Gambaryan et al. 1997). The bat IAV $\mathrm{H} 17$ and $\mathrm{H} 18 \mathrm{HA}$ proteins belong to group $1 \mathrm{HA}$ subtypes (H1, H2, H5, H6, H8, H9, H11, H12, $\mathrm{H} 13$, and $\mathrm{H} 16)$ rather than group $2(\mathrm{H} 3, \mathrm{H} 4, \mathrm{H} 7$, $\mathrm{H} 10, \mathrm{H} 14$, and H15) on a phylogenetic level (Tong et al. 2012, 2013; Wu et al. 2014) and share a remarkably high degree of structural homology with conventional IAV HA glycoproteins (Sun et al. 2013; Tong et al. 2013; Zhu et al. 2013). Furthermore, both $\mathrm{H} 17$ and H18 HAs show typical features of conventional HAs: First, a monobasic proteolytic cleavage site that can be proteolytically activated by either exogenously supplied trypsin or endogenously expressed proteases such as TMPRSS2 (Tong et al. 2013; Zhu et al. 2013; Hoffmann et al. 2016); second, the HA activation $\mathrm{pH}$ threshold necessary to form syncytia or membrane fusion is between $\mathrm{pH} 5.4$ and $\mathrm{pH} 5.6$ (Moreira et al. 2016a), which is within the typical $\mathrm{pH}$ range of most conventional HAs (Russell et al. 2018); and, third, exposure of cells to ammonium chloride potently prevented $\mathrm{H} 17$ - and $\mathrm{H} 18 \mathrm{HA}-$ mediated cell entry (Maruyama et al. 2016), showing that cell entry is indeed $\mathrm{pH}$-dependent but also proceeds through receptor-mediated endocytosis. However, despite these similarities the identified putative interface of the $\mathrm{H} 17$ and $\mathrm{H} 18 \mathrm{HA}$ receptor-binding site is strongly acidic, making binding to negatively charged sialic acid moieties rather unlikely. Consistently, neither purified H17 nor H18 HA showed a binding affinity to mammalian glycan structures in glycan microarray assays (Sun et al. 2013; Tong et al. 2013; Zhu et al. 2013). Thus, the obscure nature of these bat IAV HA proteins together with the lack of susceptible cell lines initially impeded the generation and characterization of authentic bat IAVs.
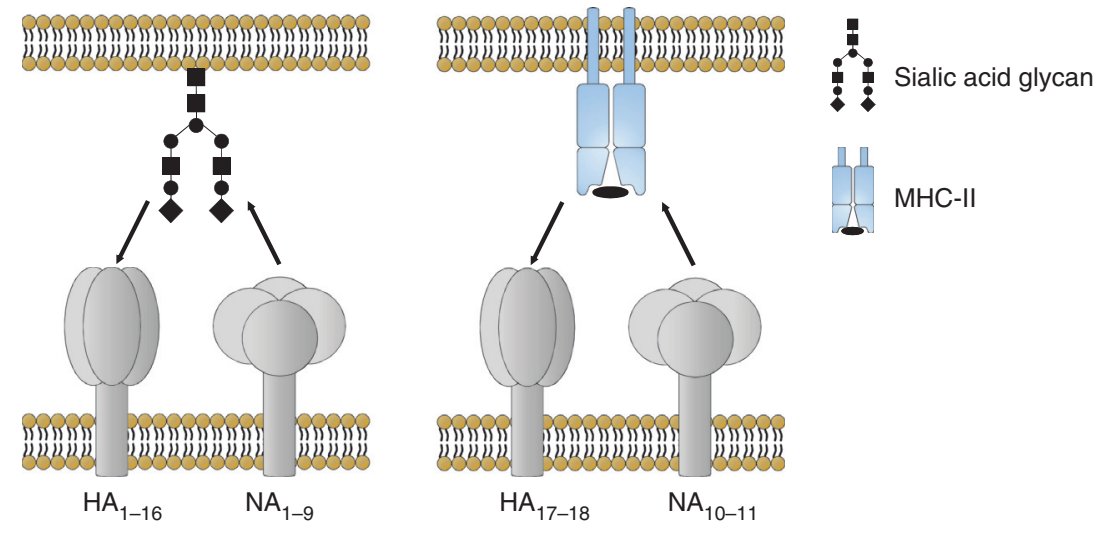

Figure 1. Current model of the receptor-binding and -modulating function of conventional and bat influenza $\mathrm{A}$ virus (IAV) surface glycoproteins. To infect a host cell, H1 to H16 hemagglutinin (HA) proteins of conventional IAV bind to sialic acid moieties exposed on cell surface glycoproteins. Neuraminidases (NAs) of the N1 to N9 subtypes facilitate viral egress by catalyzing the hydrolysis of terminal sialic acids from glycan structures. In contrast, the HA subtypes H17 and H18 use major histocompatibility complex class II (MHC-II) molecules of various species for cell entry. However, the precise H17/H18 HA-MHC-II-binding interface is currently unclear. Preliminary data suggest a MHC-II-down-regulating function for the N11 NA. Therefore, similar to conventional NA proteins, bat IAV N10 and N11 NA could counteract the H17/H18 HA function to allow virus spread. 
To overcome these obstacles, replicationcompetent vesicular stomatitis viruses (VSVs) bearing either $\mathrm{H} 17$ or H18 HA surface glycoproteins instead of the VSV-G protein were engineered. Large-scale screening with these pseudotyped viruses, solely encoding bat IAV HA proteins, revealed that, among others, the canine MDCK II and RIE1495 as well as the human U-87 and SK-Mel-28 cells were highly susceptible to infection (Moreira et al. 2016a). A combination of transcriptomic profiling of susceptible versus nonsusceptible cell lines together with a genome-wide CRISPR-Cas9based screening approach in U-87 cells eventually resulted in the identification of the human major histocompatibility complex class II (MHC-II) HLA-DR molecules as the proteinaceous receptor for both bat IAVs (Fig. 1) (Karakus et al. 2019). Using a virus-like particle approach, Giotis and colleagues confirmed HLA-DR as the receptor for H17-mediated cell entry (Giotis et al. 2019). Subsequent genetic ablation of the HLA-DR $\alpha$ chain by Karakus and colleagues (2019) rendered susceptible cells completely resistant to bat influenza virus infection, whereas ectopic expression of the HLA-DR complex in nonsusceptible cells conferred susceptibility to infection with H18N11. Moreover, expression of HLA-DR homologs from different bat species but also pigs, mice, or chickens allowed H18 HA and partially also H17 HAmediated cell entry, suggesting that both batderived influenza viruses potentially possess a broader vertebrate tropism. Interestingly, although H18 HA efficiently facilitated cell entry in cells expressing various HLA-DR homologs, H17 HA-mediated host cell entry was less effective especially in cells expressing the chicken or bat Pteropus alecto MHC-II molecules B-L and DR, respectively (Karakus et al. 2019). It remains, therefore, elusive whether H17 HA has a narrower tropism and is more restricted to its original host or alternatively relies on additional yet unknown factor(s) other than MHC-II for cell entry. Although it is assumed that $\mathrm{H} 17$ and H18 HAs physically interact with the MHC-II complex, biochemical data that show direct interaction are still missing, which is why the binding interface of these HA proteins and MHC-II is still unknown. Considering that the HA proteins of bat and conventional IAVs share presumably a common, most likely sialic acidbinding ancestor (Worobey et al. 2014), it is unclear whether the remarkable receptor switch from a "classical" glycan to an "unconventional" protein-binding interface implies an intrinsic ability of all HAs to potentially bind both protein and sialic acid receptors.

\section{BAT-DERIVED INFLUENZA VIRUSES SHOW AN UNEXPECTED ABILITY TO REPLICATE IN THE ABSENCE OF THE N11 NA SURFACE GLYCOPROTEIN}

Although all attempts to isolate infectious bat IAV from polymerase chain reaction (PCR)positive bats initially failed, the identification of susceptible cell lines eventually allowed the propagation and subsequently also the characterization of authentic bat IAVs generated by reverse genetic approaches. These viruses showed, unlike conventional IAVs, a preference to initiate infection of polarized epithelial cells from the basolateral site, whereas release of progeny virions occurred at the apical cell surface, similar to conventional IAVs (Moreira et al. 2016a). This finding can be retrospectively attributed to earlier studies that showed restricted MHC-II expression along the basolateral surface of polarized epithelial cells (Hershberg et al. 1998).

Interestingly, upon serial passaging of the bat H18N11 virus in susceptible canine RIE1495 cells or following the infection of C57BL/6 mice, mutant viruses with increased viral replication properties emerged that uniformly encoded a truncated, nonfunctional form of the N11 NA either by insertion of a premature stop codon $\left(\mathrm{N} 11_{\mathrm{G} 107 \mathrm{X}}\right)$ or by deleting the N11 vRNA sequence $\left(\mathrm{N} 11_{\text {del }}\right)$. These shortened N11 NAs encoded different portions of the transmembrane domain and parts of the stalk region but entirely lacked the ectodomain. Moreover, all of these in vitro-derived virus mutants encoded at least one additional mutation in the H18 HAs head domain, thereby enabling H18 HA-autonomous replication (Ciminski et al. 2019b). Based on these observations, it is likely that there is, similar to classical IAV surface gly- 
K. Ciminski and M. Schwemmle
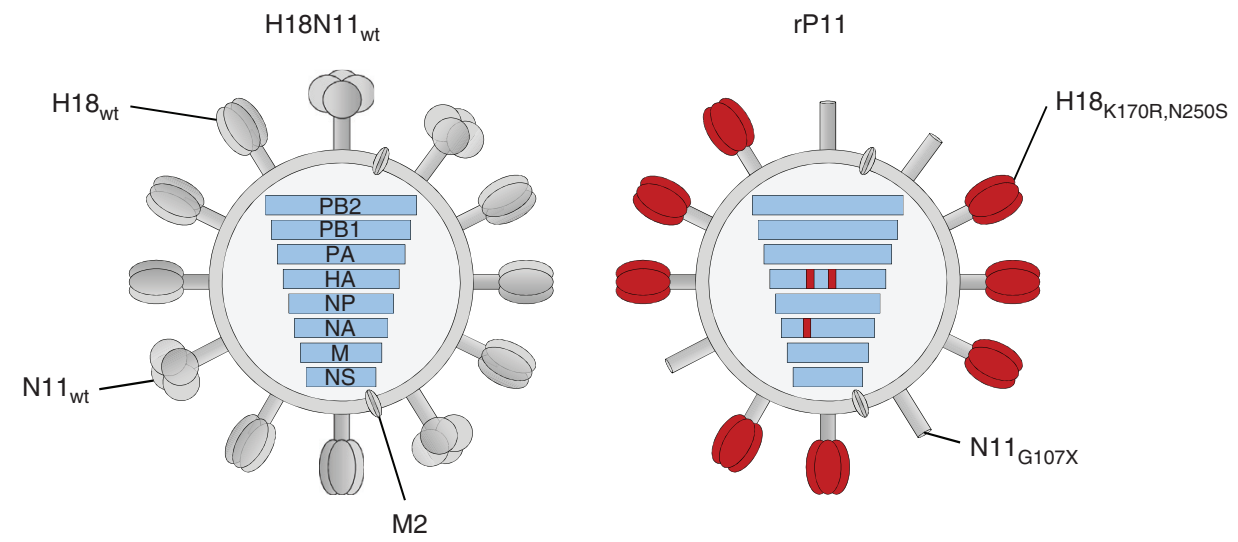

Figure 2. Mutant variants of H18N11 lack the N11 neuraminidase (NA) head domain and encode compensating mutations in H18 hemagglutinin (HA). Cartoon depicting the principle structures of the wild-type (wt) H18N11 (left) and the mutant rP11 virus particle (right). rP11 encodes the amino acid substitutions K170R and N250S located in the H18 HA head domain together with a premature stop codon in N11 at position G107X, resulting in a truncated N11 NA protein lacking the ectodomain.

coproteins, a functional cross talk between the bat IAV HA and NA proteins to balance respective activities (Kaverin et al. 1998; Mitnaul et al. 2000). Further tissue culture infection studies showed that an infectious mutant variant, designated rP11 (Fig. 2), coding for a truncated N11 NA $\left(\mathrm{N} 11_{\mathrm{G} 107 \mathrm{X}}\right)$ together with the two amino acid substitutions K170R and N250S in the H18 HA head domain $\left(\mathrm{H}_{1} 8_{\mathrm{K} 170 \mathrm{R}, \mathrm{N} 250 \mathrm{~S}}\right)$ still relied on MHC-II surface expression for host cell entry. However, in contrast to wild-type (wt) $\mathrm{H} 18 \mathrm{~N} 11, \mathrm{rP} 11$ replicated to higher titers in vitro and also in mice. Although an explanation for the selection of truncated N11 NA is still lacking, it is tempting to speculate that the negatively charged N11 NA head domain might disturb proper H18 HA-mediated entry caused by repulsions with the also negatively charged cellular membrane. Importantly, even though NA-negative mutants have been described for classical IAVs before, the ability of H18N11 to compensate for the lack of a functional NA without impaired viral growth in vitro and in vivo is unprecedented among all other known classical subtypes of influenza (Hughes et al. 2000; Kalthoff et al. 2013; Samson et al. 2014; Ann et al. 2016). Whether H17N10 possesses a comparable N10 NA-independent replication capacity remains to be determined.

\section{DO BAT IAV NA PROTEINS COUNTERACT THE BAT IAV HA FUNCTION?}

To counteract the sialic acid-binding function of HA, conventional IAVs encode a second surface glycoprotein: NA. The function of this type II membrane protein is essential for virus transmission as its sialidase activity catalyzes hydrolysis of sialic acid residues from glycan structures, thereby facilitating viral particle release from infected host cells (Fig. 1) (Air and Laver 1989). Based on their phylogenetic relationships, conventional NA proteins fall into either group $1(\mathrm{~N} 1, \mathrm{~N} 4, \mathrm{~N} 5$, and N8) or group 2 (N2, N3, N6, N7, and N9). In contrast, the bat IAV N10 and N11 NA proteins show an extensive divergence from all known influenza NA proteins and lack the canonical sialidase function because of conformational changes in the putative active pocket and the substitution of key amino acid residues that are required for NA activity (Li et al. 2012; Tong et al. 2012, 2013; Zhu et al. 2012). Therefore, the new N10 and N11 NA subtypes were proposed (Wu et al. 2014). Most interestingly, despite $<30 \%$ of amino acid sequence homology with conventional NAs (Tong et al. 2012, 2013), the bat IAV NA proteins form the typical homotetrameric "boxshaped" complex with each monomer contain- 
ing a propeller-like structure of six antiparallel $\beta$ sheets in the head domain ( $\mathrm{Li}$ et al. 2012; Zhu et al. 2012; Tong et al. 2013) and possessing the highly conserved calcium ion-binding site that is required for thermostability and activity of all known influenza A and B NA active sites (Dimmock 1971; Chong et al. 1991; Burmeister et al. 1994; Smith et al. 2006). Although the specificity and function of the bat IAV NA proteins remain elusive, a modeling study predicted a strong structural concordance between the staphylococcal enterotoxin I (SEI) domain, known to bind the human MHC-II $\beta$-chain, and the N10 NA head domain, suggesting that N10 NA might interact with MHC-II molecules (Weininger and Weininger 2015). Similarly, the extracellular N11 NA head domain shares structural similarity with multiple bacterial toxins including SEI (Weininger and Weininger 2015). The first evidence for the function of N11 NA in the viral life cycle of H18N11 came from infection experiments of human HLA-DR MHC-II overexpressing cells with different recombinant H18N11 variants encoding combinations of $\mathrm{H} 18 \mathrm{HA}\left(\mathrm{H} 18_{\mathrm{wt}}\right.$ or $\left.\mathrm{H} 18_{\mathrm{K} 170 \mathrm{R}, \mathrm{N} 250 \mathrm{~S}}\right)$ together with either functional $\left(\mathrm{N} 11_{\mathrm{wt}}\right)$ or a truncated version of N11 NA. Interestingly, although all recombinant virus variants were initially infectious, propagation of viruses encoding $\mathrm{H}_{18} \mathrm{wA}$ plus a truncated N11 NA was not possible, suggesting that full-length N11 NA is required in combination with $\mathrm{H} 18_{\mathrm{wt}}$. In sharp contrast, all viruses harboring $\mathrm{H} 18_{\mathrm{K} 170 \mathrm{R}, \mathrm{N} 250 \mathrm{~S}}$ were infectious and replicated to comparable titers regardless of whether full-length N11 NA was present, showing that H18 K170R,N250s $_{\text {enables N11 NA-independent }}$ viral replication. Finally, immunostaining of wt H18N11-infected MHC-II-expressing cells revealed reduced levels of total MHC-II levels compared with cells infected with a mutant variant encoding $\mathrm{H} 18_{\mathrm{K} 170 \mathrm{R}, \mathrm{N} 250 \mathrm{~S}}$ and $\mathrm{N} 11_{\mathrm{G} 107 \mathrm{X}}$, suggesting a role of full-length N11 NA in the down-regulation of MHC-II expression (Ciminski et al. 2019b). Thus, although the precise function and mode of action of N11 NA is still unclear, it is tempting to speculate that the NA protein counteracts H18 HA-mediated receptor binding to facilitate particle release. Conse- quently, N11 NA would act in a similar manner to classical NA proteins that possess a receptor destroying function (Fig. 1). There are currently no experimental data available for N10 NA, but considering that H17 HA also uses MHC-II for cell entry, N10 NA may also have a MHC-II down-regulating function.

\section{H18N11 REPLICATES EFFICIENTLY IN ITS NATURAL HOSTS}

Serosurveys indicate that several South American bat species can be infected with bat IAV (Tong et al. 2013), but due to the lack of acutely infected animals, the precise organ tropism, and the route of bat-to-bat transmission remained unclear. Infection studies with wt H18N11 in the Neotropical Jamaican fruit bat (Artibeus jamaicensis), a close relative of the initially identified host species Artibeus planirostris and a likely reservoir host, showed that intranasally inoculated bats are susceptible to bat IAV infection and subsequently shed high loads of infectious wt influenza virus via the rectal route, eventually resulting in a horizontal transmission to naive contact bats (Ciminski et al. 2019b). Accordingly, in these infected bats, H18N11 RNA and antigen were predominantly detected in the follicle-associated epithelium (FAE) of jejunal Peyer's patches, a part of the gut-associated lymphoid tissue, and also in the squamous epithelium of the palatine tonsils-both being highly immunocompetent compartments occupied by a high number of lymphoid cells (Fig. 3). Intriguingly, none of the histopathologically investigated organs showed necrotizing or inflammatory lesions, in line with the previously described disease tolerance of bats as a reservoir host, resulting in asymptomatic infections of most of their associated viruses (Mandl et al. 2015; Schountz et al. 2017). The finding that viral replication occurred in the FAE of the gut-associated lymphoid tissue led to the assumption that the virus might be taken up from the gastrointestinal lumen similar to low pathogenic avian IAVs that traverse the digestive tract of ducks and replicate in the intestine before being excreted at high concentrations in feces (Webster et al. 1978). Importantly, inocu- 


\section{K. Ciminski and M. Schwemmle}
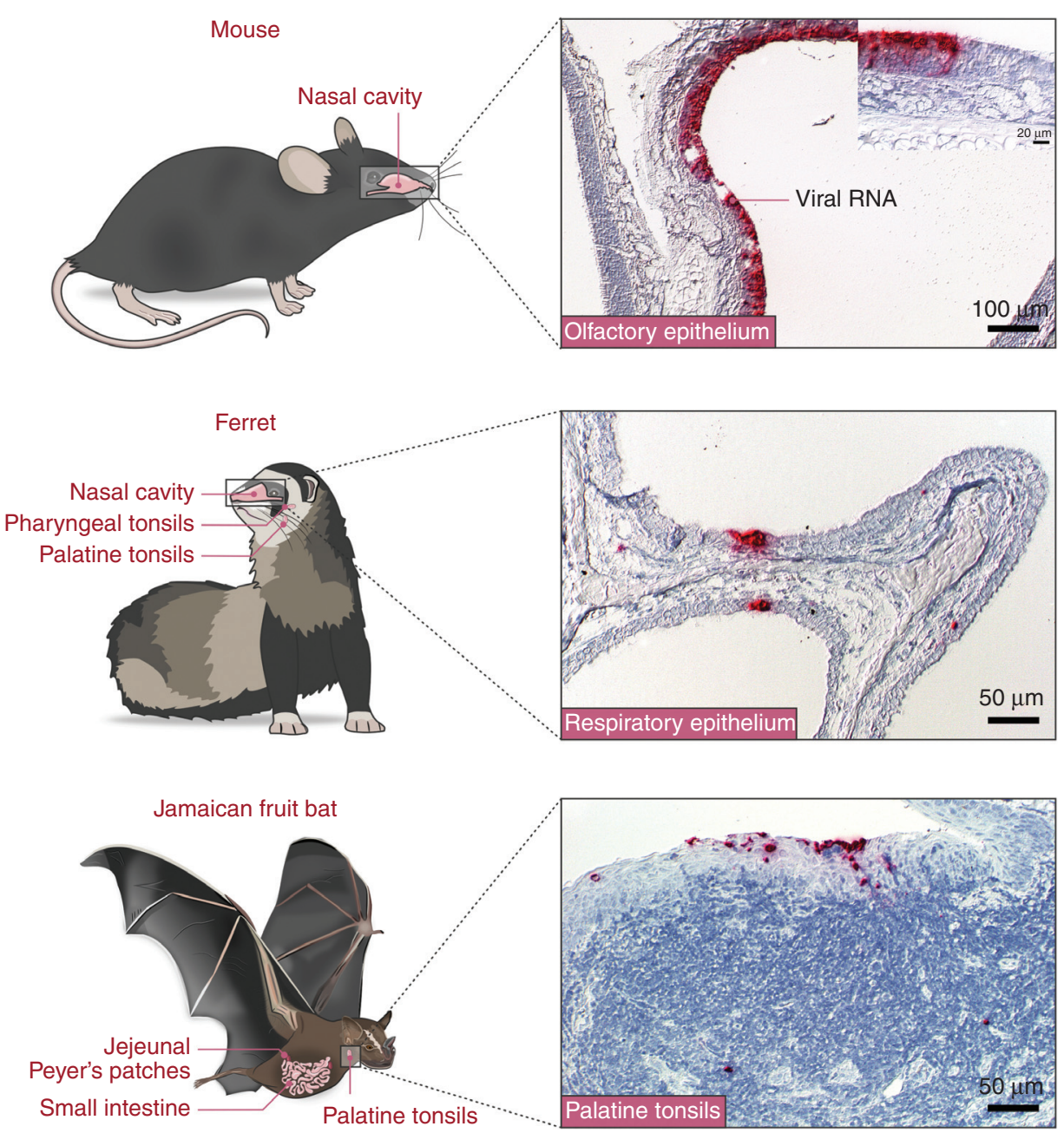

Figure 3. Tissue tropism of H18N11 viruses in different mammalian species following intranasal inoculation. In mice, H18N11 replicates exclusively in the olfactory epithelium of the nasal cavity; in ferrets, especially the respiratory epithelium of the nasal cavity and the follicle-associated epithelium of the pharyngeal as well as the palatine tonsils, were identified as the sites of viral replication. In Jamaican fruit bats, H18N11 was found in the squamous epithelium of the palatine tonsils, the lamina propria of the small intestine, and the follicleassociated epithelium of the jejunal Peyer's patches. Representative images showing the detection of H18-specifc viral RNA for selected infected organs from either mice, ferrets, and Jamaican fruit bats are shown on the right. The magnification is indicated. (Images provided by Reiner Ulrich and Jan Schinköthe, Institute of Veterinary Pathology, Leipzig University.)

lation of the mutant $\mathrm{rP} 11$ resulted in a productive infection of Jamaican fruit bats, which then shed infectious virus in the feces (Ciminski et al. $2019 b$ ). Sequencing of the isolated viral content from index animals revealed that both substitutions at K170R and N250S in H18 HA were maintained, whereas (partial) restoration of the N11 open reading frame was selected. Furthermore, the fact that only virus variants encoding a full-length N11 NA were transmitted to naive contact bats suggests a strong selection pressure on a presumably functional N11 NA during viral replication in lymphoid tissues and for virus spread among bats. 


\section{H18N11 IS ONLY POORLY ADAPTED TO NON-BAT SPECIES}

Classical influenza viruses originating from aquatic waterfowl circulate in a broad range of different hosts, which not only drives the virus' evolution owing to host-specific adaptations but is also a prerequisite for its overall maintenance. In contrast, evaluation of the bat IAV host spectrum is still in its early stages, and it is unclear whether other animals besides bats are naturally infected. Importantly, studies to determine the prevalence of $\mathrm{H} 17 / \mathrm{H} 18 \mathrm{HA}$-specific antibodies in non-bat species in Central and South America are missing. Recent experimental infection studies, however, provided first insights into the ability of bat IAV to replicate in other mammals such as mice and ferrets. Following intranasal inoculation of mice with either wt H18N11 or the mutant variant $\mathrm{rP} 11$, viral replication was exclusively constrained to the olfactory epithelium of the upper respiratory tract (URT) without causing any signs of disease (Fig. 3) (Ciminski et al. 2019b). Moreover, despite robust viral replication in the URT, no transmission among immunocompromised mice lacking the type I and III interferon (IFN) receptor was observed. Interestingly, although rP11 appeared genetically stable in mice and maintained the inserted mutations at $\mathrm{H} 18_{\mathrm{K} 170 \mathrm{R}, \mathrm{N} 250 \mathrm{~S}}$ and $\mathrm{N} 11_{\mathrm{G} 107 \mathrm{X}}$, some of the viruses isolated from wt H18N11-infected animals carried a $\mathrm{N} 11_{\text {del }}$ gene segment, suggesting that in mice, similar to tissue culture, efficient viral growth is facilitated by a loss of full-length N11 NA. In ferrets, intranasal administration of wt $\mathrm{H} 18 \mathrm{~N} 11$ resulted in no detectable virus growth in any investigated organ, but provoked seroconversion with H18 HAspecific neutralizing antibodies. In contrast, following inoculation with rP11, H18N11 RNA was found in various organs including the URT, the lung, and the brains of some of the infected animals (Ciminski et al. 2019b). Most interestingly, histopathological analysis of the infected ferrets revealed productive infection of the FAE of the pharyngeal tonsils (Fig. 3). Given that MHC-II is the entry receptor, this observation could indicate that MHC-II-expressing antigen-presenting cells are the preferred host cells for bat IAVs. Consistent with this tropism and the above described putative MHC-II down-regulating function of N11 NA, all isolated viral sequences showed a partial or even entire restoration of the N11 vRNA, but maintained both mutations in H18 HA. Similar to mice, infected ferrets appeared clinically healthy and did not transmit the virus to naive contact animals, suggesting that bat IAVs are only poorly adapted to non-bat species.

\section{IS THERE A ZOONOTIC RISK?}

For the evaluation of the zoonotic risk of conventional influenza viruses, ferrets are the favored animal model to study human IAV infections because they express $\alpha 2,6$-linked $N$ acetylneuraminic acids in the respiratory tract and hence most closely reflect influenza virus transmissibility and the resulting pathogenicity in humans (Maher and DeStefano 2004; Belser et al. 2011; Moore et al. 2014). The poor replication properties of wt $\mathrm{H} 18 \mathrm{~N} 11$ and the rP11 variant together with the absence of viral transmission between ferrets may suggest low zoonotic potential of bat influenza viruses. However, the fact that both $\mathrm{H} 17 \mathrm{~N} 10$ and H18N11 use MHC-II molecules instead of sialic acid residues challenges the predictive value of this animal model with regard to the zoonotic potential for humans. Furthermore, it is currently not known whether humans and ferrets share a similar MHC-II receptor distribution and how efficient ferret MHC-II might support H17-and H18 HA-mediated host cell entry.

Another important aspect for the zoonotic potential of influenza viruses and their ability to establish a lineage in the human population is whether they are able to overcome host restriction factors such as Mx1 proteins (in humans $\mathrm{MxA}) . \mathrm{MxA}$ is a type I and III IFN-induced large dynamin-like GTPase that strongly suppresses viral replication at an early stage of the viral life cycle. In fact, all known human-adapted IAV strains encode specific adaptive mutations in NP, facilitating MxA escape (Mänz et al. 2013; Ashenberg et al. 2017). Importantly, the 2009 pandemic virus as well as the recently isolated Eurasian avian-like swine IAV highlight that 
these adaptive mutations can be acquired in functional Mxl-expressing intermediate hosts like swine (Mänz et al. 2013; Dornfeld et al. 2018). Intriguingly, the Mx1 proteins from Sturnira lilium and Carollia perspicillata, both bat influenza host species (Tong et al. 2012, 2013), exert antiviral activity (Fuchs et al. 2017). Nevertheless, bat IAV NPs, in contrast to humanadapted IAV NPs, lack known MxA escape mutations (Ciminski et al. 2019a). Consequently, the human restriction factor MxA was found to efficiently restrict replication of a chimeric bat virus PR8-H18N11 in both MxA-expressing cell lines and in a MxA transgenic mouse model, indicating that H18N11 is currently unable to overcome the human MxA interspecies barrier (Ciminski et al. 2019a).

\section{CONCLUDING REMARKS}

The discovery of the two novel influenza subtypes $\mathrm{H} 17 \mathrm{~N} 10$ and $\mathrm{H} 18 \mathrm{~N} 11$ circulating in bats paved the way for a new era in IAV research by challenging the dogma that all known influenza viruses emerge from aquatic waterfowl and use sialic acid receptors. Although it is still unclear why, we now know that the bat IAV HA homologs underwent a switch in specificity to use a proteinaceous instead of a sialic acid receptor. Concurrently, the bat IAV NA proteins acquired a new, likely MHC-II receptor-modulating function. Moreover, by studying and comparing NPs of both classical and bat IAVs, a new aspect of genome packaging was identified: Aside from specific vRNA sequences, genome packaging is also driven by a finetuned NP amino acid sequence. Together, these differences are the consequence of a divergent evolution of IAVs in bat species compared with other hosts, highlighting that influenza virus plasticity is far from being entirely understood. Therefore, further studies of the bat IAVs are poised to unveil additional novel mechanisms regarding the viral life cycle and virus-host interactions. Especially, the recently established Jamaican fruit bat infection model will help to understand the dampened innate immune response, which make bats an excellent host reservoir for viruses.

\section{ACKNOWLEDGMENTS}

The authors acknowledge all the people who have contributed to understanding and deciphering the bat-derived influenza viruses over the years in our and our partner's laboratories. We thank Reiner Ulrich and Jan Schinköthe for providing histopathological images, Hardin Bolte for the generation of figures, and Sebastian Giese and Geraldine Goh for critically reading the manuscript. This work was supported by a grant from the Deutsche Forschungsgemeinschaft (DFG) to M.S. (SCHW 632/17-2).

This article has been made freely available online courtesy of TAUNS Laboratories.

\section{REFERENCES}

Air GM, Laver WG. 1989. The neuraminidase of influenza virus. Proteins 6: 341-356. doi:10.1002/prot .340060402

Ann J, Abed Y, Beaulieu E, Bouhy X, Joly MH, Dubé K, Carbonneau J, Hamelin ME, Mallett C, Boivin G. 2016. Impact of a large deletion in the neuraminidase protein identified in a laninamivir-selected influenza A/Brisbane/ $10 / 2007$ (H3N2) variant on viral fitness in vitro and in ferrets. Influenza Other Respir Viruses 10: 122-126. doi:10.1111/irv.12356

Ashenberg O, Padmakumar J, Doud MB, Bloom JD. 2017. Deep mutational scanning identifies sites in influenza nucleoprotein that affect viral inhibition by $\mathrm{MxA}$ PLoS Pathog 13: e1006288. doi:10.1371/journal.ppat .1006288

Belser JA, Katz JM, Tumpey TM. 2011. The ferret as a model organism to study influenza A virus infection. Dis Model Mech 4: 575-579. doi:10.1242/dmm.007823

Brunotte L, Beer M, Horie M, Schwemmle M. 2016. Chiropteran influenza viruses: flu from bats or a relic from the past? Curr Opin Virol 16: 114-119. doi:10.1016/j.coviro .2016 .02 .003

Burmeister WP, Cusack S, Ruigrok RW. 1994. Calcium is needed for the thermostability of influenza $B$ virus neuraminidase. J Gen Virol 75: 381-388. doi:10.1099/00221317-75-2-381

Calisher CH, Childs JE, Field HE, Holmes KV, Schountz T. 2006. Bats: important reservoir hosts of emerging viruses. Clin Microbiol Rev 19: 531-545. doi:10.1128/CMR .00017-06

Campos ACA, Góes LGB, Moreira-Soto A, de Carvalho C, Ambar G, Sander AL, Fischer C, da Rosa AR, de Oliveira DC, Kataoka APG, et al. 2019. Bat influenza A (HL18NL11) virus in fruit bats, Brazil. Emerg Infect Dis 25: 333-337. doi:10.3201/eid2502.181246

Chong AK, Pegg MS, von Itzstein M. 1991. Influenza virus sialidase: effect of calcium on steady-state kinetic parameters. Biochim Biophys Acta 1077: 65-71. doi:10.1016/ 0167-4838(91)90526-6 
Ciminski K, Thamamongood T, Zimmer G, Schwemmle M 2017. Novel insights into bat influenza A viruses. J Gen Virol 98: 2393-2400. doi:10.1099/jgv.0.000927

Ciminski K, Pulvermüller J, Adam J, Schwemmle M. 2019a. Human MxA is a potent interspecies barrier for the novel bat-derived influenza A-like virus H18N11. Emerg Microbes Infect 8: 556-563. doi:10.1080/22221751.2019 .1599301

Ciminski K, Ran W, Gorka M, Lee J, Malmlov A, Schinköthe J, Eckley M, Murrieta RA, Aboellail TA, Campbell CL, et al. 2019b. Bat influenza viruses transmit among bats but are poorly adapted to non-bat species. Nat Microbiol doi:10.1038/s41564-019-0556-9

Compans RW, Content J, Duesberg PH. 1972. Structure of the ribonucleoprotein of influenza virus. J Virol 10: 795800 .

Dimmock NJ. 1971. Dependence of the activity of an influenza virus neuraminidase upon $\mathrm{Ca}^{2+}$. J Gen Virol 13: 481483. doi:10.1099/0022-1317-13-3-481

Dornfeld D, Petric PP, Hassan E, Zell R, Schwemmle M. 2018. Eurasian avian-like swine influenza A viruses escape human MxA restriction through distinct mutations in their nucleoprotein. J Virol. 93: e00997-18. doi:10 1128/JVI.00997-18

Fereidouni S, Kwasnitschka L, Balkema Buschmann A, Müller T, Freuling C, Schatz J, Pikula J, Bandouchova H, Hoffmann R, Ohlendorf B, et al. 2015. No virological evidence for an influenza A-like virus in European bats. Zoonoses Public Health 62: 187-189. doi:10.1111/zph .12131

Fodor E, Seong BL, Brownlee GG. 1993. Photochemical cross-linking of influenza A polymerase to its virion RNA promoter defines a polymerase binding site at residues 9 to 12 of the promoter. J Gen Virol 74: 1327-1333. doi:10.1099/0022-1317-74-7-1327

Freidl GS, Binger T, Müller MA, de Bruin E, van Beek J, Corman VM, Rasche A, Drexler JF, Sylverken A, Oppong SK, et al. 2015. Serological evidence of influenza A viruses in frugivorous bats from Africa. PLoS ONE 10: 0127035. doi:10.1371/journal.pone.0127035

Fuchs J, Hölzer M, Schilling M, Patzina C, Schoen A, Hoenen T, Zimmer G, Marz M, Weber F, Müller MA, et al 2017. Evolution and antiviral specificities of interferoninduced $\mathrm{Mx}$ proteins of bats against ebola, influenza, and other RNA viruses. J Virol 91: e00361-17. doi:10.1128/JVI .00361-17

Fujii Y, Goto H, Watanabe T, Yoshida T, Kawaoka Y. 2003. Selective incorporation of influenza virus RNA segments into virions. Proc Natl Acad Sci 100: 2002-2007. doi:10 $.1073 /$ pnas.0437772100

Gambaryan AS, Tuzikov AB, Piskarev VE, Yamnikova SS, Lvov DK, Robertson JS, Bovin NV, Matrosovich MN 1997. Specification of receptor-binding phenotypes of influenza virus isolates from different hosts using synthetic sialylglycopolymers: non-egg-adapted human $\mathrm{H} 1$ and $\mathrm{H} 3$ influenza A and influenza B viruses share a common high binding affinity for $6^{\prime}$-sialyl( $N$-acetyllactosamine). Virology 232: 345-350. doi:10.1006/viro.1997.8572

Giotis ES, Carnell G, Young EF, Ghanny S, Soteropoulos P, Wang LF, Barclay WS, Skinner MA, Temperton N. 2019. Entry of the bat influenza H17N10 virus into mammalian cells is enabled by the MHC class II HLA-DR receptor. Nat Microbiol doi:10.1038/s41564-019-0517-3

Goto H, Muramoto Y, Noda T, Kawaoka Y. 2013. The genome-packaging signal of the influenza A virus genome comprises a genome incorporation signal and a genomebundling signal. J Virol 87: 11316-11322. doi:10.1128/JVI .01301-13

Hayman DT. 2016. Bats as viral reservoirs. Annu Rev Virol 3: 77-99. doi:10.1146/annurev-virology-110615-042203

Hershberg RM, Cho DH, Youakim A, Bradley MB, Lee JS, Framson PE, Nepom GT. 1998. Highly polarized HLA class II antigen processing and presentation by human intestinal epithelial cells. J Clin Invest 102: 792-803. doi:10.1172/JCI3201

Hoffmann M, Krüger N, Zmora P, Wrensch F, Herrler G, Pöhlmann S. 2016. The hemagglutinin of bat-associated influenza viruses is activated by TMPRSS2 for $\mathrm{pH}$-dependent entry into bat but not human cells. PLOS ONE 11: e0152134. doi:10.1371/journal.pone.0152134

Hsu MT, Parvin JD, Gupta S, Krystal M, Palese P. 1987. Genomic RNAs of influenza viruses are held in a circular conformation in virions and in infected cells by a terminal panhandle. Proc Natl Acad Sci 84: 8140-8144. doi:10 $.1073 /$ pnas.84.22.8140

Hughes MT, Matrosovich M, Rodgers ME, McGregor M, Kawaoka Y. 2000. Influenza A viruses lacking sialidase activity can undergo multiple cycles of replication in cell culture, eggs, or mice. J Virol 74: 5206-5212. doi:10 $.1128 / J V I .74 .11 .5206-5212.2000$

Hutchinson EC, von Kirchbach JC, Gog JR, Digard P. 2010. Genome packaging in influenza A virus. J Gen Virol 91: 313-328. doi:10.1099/vir.0.017608-0

Johnson KEE, Song T, Greenbaum B, Ghedin E. 2017. Getting the flu: 5 key facts about influenza virus evolution. PLoS Pathog 13: e1006450. doi:10.1371/journal.ppat .1006450

Juozapaitis M, Aguiar Moreira E, Mena I, Giese S, Riegger D, Pohlmann A, Höper D, Zimmer G, Beer M, García-Sastre A, et al. 2014. An infectious bat-derived chimeric influenza virus harbouring the entry machinery of an influenza A virus. Nat Commun 5: 4448. doi:10.1038/ncomms 5448

Kalthoff D, Rohrs S, Hoper D, Hoffmann B, Bogs J, Stech J, Beer M. 2013. Truncation and sequence shuffling of segment 6 generate replication-competent neuraminidase-negative influenza $\mathrm{H} 5 \mathrm{~N} 1$ viruses. J Virol 87: 13556-13568. doi:10.1128/JVI.02244-13

Kandeil A, Gomaa MR, Shehata MM, El Taweel AN, Mahmoud SH, Bagato O, Moatasim Y, Kutkat O, Kayed AS, Dawson P, et al. 2019. Isolation and characterization of a distinct influenza A virus from Egyptian bats. J Virol 93: e01059-18. doi:10.1128/JVI.01059-18

Karakus U, Thamamongood T, Ciminski K, Ran W, Günther SC, Pohl MO, Eletto D, Jeney C, Hoffmann D, Reiche S, et al. 2019. MHC class II proteins mediate cross-species entry of bat influenza viruses. Nature 567: 109-112. doi:10.1038/s41586-019-0955-3

Kaverin NV, Gambaryan AS, Bovin NV, Rudneva IA, Shilov AA, Khodova OM, Varich NL, Sinitsin BV, Makarova NV, Kropotkina EA. 1998. Postreassortment changes in influenza A virus hemagglutinin restoring HA-NA func- 
K. Ciminski and M. Schwemmle

tional match. Virology 244: 315-321. doi:10.1006/viro .1998 .9119

Li Q, Sun X, Li Z, Liu Y, Vavricka CJ, Qi J, Gao GF. 2012. Structural and functional characterization of neuraminidase-like molecule N10 derived from bat influenza A virus. Proc Natl Acad Sci 109: 18897-18902. doi:10.1073/ pnas.1211037109

Maher JA, DeStefano J. 2004. The ferret: an animal model to study influenza virus. Lab Anim 33: 50-53. doi:10.1038/ laban1004-50

Mandl JN, Ahmed R, Barreiro LB, Daszak P, Epstein JH, Virgin HW, Feinberg MB. 2015. Reservoir host immune responses to emerging zoonotic viruses. Cell 160: 20-35. doi:10.1016/j.cell.2014.12.003

Mänz B, Dornfeld D, Götz V, Zell R, Zimmermann P, Haller O, Kochs G, Schwemmle M. 2013. Pandemic influenza A viruses escape from restriction by human MxA through adaptive mutations in the nucleoprotein. PLoS Pathog 9: e1003279. doi:10.1371/journal.ppat.1003279

Maruyama J, Nao N, Miyamoto H, Maeda K, Ogawa H, Yoshida R, Igarashi M, Takada A. 2016. Characterization of the glycoproteins of bat-derived influenza viruses. Virology 488: 43-50. doi:10.1016/j.virol.2015.11.002

Mitnaul LJ, Matrosovich MN, Castrucci MR, Tuzikov AB, Bovin NV, Kobasa D, Kawaoka Y. 2000. Balanced hemagglutinin and neuraminidase activities are critical for efficient replication of influenza A virus. $J$ Virol 74: 6015-6020. doi:10.1128/JVI.74.13.6015-6020.2000

Moore IN, Lamirande EW, Paskel M, Donahue D, Kenney H, Qin J, Subbarao K. 2014. Severity of clinical disease and pathology in ferrets experimentally infected with influenza viruses is influenced by inoculum volume. J Virol $\mathbf{8 8}$ : 13879-13891. doi:10.1128/JVI.02341-14

Moreira EA, Locher S, Kolesnikova L, Bolte H, Aydillo T, García-Sastre A, Schwemmle M, Zimmer G. 2016a. Synthetically derived bat influenza A-like viruses reveal a cell type- but not species-specific tropism. Proc Natl Acad Sci 113: 12797-12802. doi:10.1073/pnas.1608821113

Moreira EA, Weber A, Bolte H, Kolesnikova L, Giese S, Lakdawala S, Beer M, Zimmer G, García-Sastre A, Schwemmle M, et al. 2016b. A conserved influenza A virus nucleoprotein code controls specific viral genome packaging. Nat Commun 7: 12861. doi:10.1038/ncomms 12861

Olival KJ, Hosseini PR, Zambrana-Torrelio C, Ross N, Bogich TL, Daszak P. 2017. Host and viral traits predict zoonotic spillover from mammals. Nature 546: 646650. doi:10.1038/nature22975

Palese P, Schulman JL. 1976. Mapping of the influenza virus genome: identification of the hemagglutinin and the neuraminidase genes. Proc Natl Acad Sci 73: 2142 2146. doi:10.1073/pnas.73.6.2142

Pflug A, Guilligay D, Reich S, Cusack S. 2014. Structure of influenza A polymerase bound to the viral RNA promoter. Nature 516: 355-360. doi:10.1038/nature14008

Poole DS, Yu S, Cai Y, Dinis JM, Muller MA, Jordan I, Friedrich TC, Kuhn JH, Mehle A. 2014. Influenza A virus polymerase is a site for adaptive changes during experimental evolution in bat cells. J Virol 88: 12572-12585. doi:10.1128/JVI.01857-14
Russell CJ, Hu M, Okda FA. 2018. Influenza hemagglutinin protein stability, activation, and pandemic risk. Trends Microbiol 26: 841-853. doi:10.1016/j.tim.2018.03.005

Samson M, Abed Y, Desrochers FM, Hamilton S, Luttick A, Tucker SP, Pryor MJ, Boivin G. 2014. Characterization of drug-resistant influenza virus $\mathrm{A}(\mathrm{H} 1 \mathrm{~N} 1)$ and $\mathrm{A}(\mathrm{H} 3 \mathrm{~N} 2)$ variants selected in vitro with laninamivir. Antimicrob Agents Chemother 58: 5220-5228. doi:10.1128/AAC .03313-14

Sauter NK, Bednarski MD, Wurzburg BA, Hanson JE, Whitesides GM, Skehel JJ, Wiley DC. 1989. Hemagglutinins from two influenza virus variants bind to sialic acid derivatives with millimolar dissociation constants: a 500 $\mathrm{MHz}$ proton nuclear magnetic resonance study. Biochemistry 28: 8388-8396. doi:10.1021/bi00447a018

Schountz T, Baker ML, Butler J, Munster V. 2017. Immunological control of viral infections in bats and the emergence of viruses highly pathogenic to humans. Front Immunol 8: 1098. doi:10.3389/fimmu.2017.01098

Smith BJ, Huyton T, Joosten RP, McKimm-Breschkin JL, Zhang JG, Luo CS, Lou MZ, Labrou NE, Garrett TP. 2006. Structure of a calcium-deficient form of influenza virus neuraminidase: implications for substrate binding. Acta Crystallogr D Biol Crystallogr 62: 947-952. doi:10 .1107/S0907444906020063

Sun X, Shi Y, Lu X, He J, Gao F, Yan J, Qi J, Gao GF. 2013. Bat-derived influenza hemagglutinin H17 does not bind canonical avian or human receptors and most likely uses a unique entry mechanism. Cell Rep 3: 769-778. doi:10 .1016/j.celrep.2013.01.025

Tong S, Li Y, Rivailler P, Conrardy C, Castillo DA, Chen LM, Recuenco S, Ellison JA, Davis CT, York IA, et al. 2012. A distinct lineage of influenza A virus from bats. Proc Natl Acad Sci 109: 4269-4274. doi:10.1073/pnas .1116200109

Tong S, Zhu X, Li Y, Shi M, Zhang J, Bourgeois M, Yang H, Chen X, Recuenco S, Gomez J, et al. 2013. New world bats harbor diverse influenza A viruses. PLoS Pathog 9: e1003657. doi:10.1371/journal.ppat.1003657

Watanabe T, Watanabe S, Noda T, Fujii Y, Kawaoka Y. 2003. Exploitation of nucleic acid packaging signals to generate a novel influenza virus-based vector stably expressing two foreign genes. J Virol 77: 10575-10583. doi:10.1128/JVI .77.19.10575-10583.2003

Webster RG, Yakhno M, Hinshaw VS, Bean WJ, Murti KG. 1978. Intestinal influenza: replication and characterization of influenza viruses in ducks. Virology 84: 268-278. doi:10.1016/0042-6822(78)90247-7

Webster RG, Bean WJ, Gorman OT, Chambers TM, Kawaoka Y. 1992. Evolution and ecology of influenza A viruses. Microbiol Rev 56: 152-179.

Weininger A, Weininger S. 2015. Using common spatial distributions of atoms to relate functionally divergent influenza virus N10 and N11 protein structures to functionally characterized neuraminidase structures, toxin cell entry domains, and non-influenza virus cell entry domains. PLoS ONE 10: e0117499. doi:10.1371/journal .pone.0117499

White MC, Lowen AC. 2018. Implications of segment mismatch for influenza A virus evolution. J Gen Virol 99: 3-16. doi:10.1099/jgv.0.000989 
Worobey M, Han GZ, Rambaut A. 2014. A synchronized global sweep of the internal genes of modern avian influenza virus. Nature 508: 254-257. doi:10.1038/nature 13016

Wu Y, Wu Y, Tefsen B, Shi Y, Gao GF. 2014. Bat-derived influenza-like viruses $\mathrm{H} 17 \mathrm{~N} 10$ and H18N11. Trends Microbiol 22: 183-191. doi:10.1016/j.tim.2014.01.010

Yang J, Lee J, Ma J, Lang Y, Nietfeld J, Li Y, Duff M, Li Y, Yang Y, Liu H, et al. 2017. Pathogenicity of modified bat influenza virus with different $M$ genes and its reassortment potential with swine influenza A virus. J Gen Virol 98: 577-584. doi:10.1099/jgv.0.000715

Zhao X, Tefsen B, Li Y, Qi J, Lu G, Shi Y, Yan J, Xiao H, Gao GF. 2016. The NS1 gene from bat-derived influenza-like virus $\mathrm{H} 17 \mathrm{~N} 10$ can be rescued in influenza A PR8 backbone. J Gen Virol 97: 1797-1806. doi:10.1099/jgv.0 .000509
Zhou B, Ma J, Liu Q, Bawa B, Wang W, Shabman RS, Duff M, Lee J, Lang Y, Cao N, et al. 2014. Characterization of uncultivable bat influenza virus using a replicative synthetic virus. PLoS Pathog 10: e1004420. doi:10.1371/jour nal.ppat. 1004420

Zhu X, Yang H, Guo Z, Yu W, Carney PJ, Li Y, Chen LM, Paulson JC, Donis RO, Tong S, et al. 2012. Crystal structures of two subtype N10 neuraminidase-like proteins from bat influenza A viruses reveal a diverged putative active site. Proc Natl Acad Sci 109: 18903-18908. doi:10 $.1073 /$ pnas.1212579109

Zhu X, Yu W, McBride R, Li Y, Chen LM, Donis RO, Tong S, Paulson JC, Wilson IA. 2013. Hemagglutinin homologue from H17N10 bat influenza virus exhibits divergent receptor-binding and $\mathrm{pH}$-dependent fusion activities. Proc Natl Acad Sci 110: 1458-1463. doi:10.1073/pnas .1218509110 


\section{$\&_{\mathrm{CSH}}^{\infty} \&$ Cold Spring Harbor

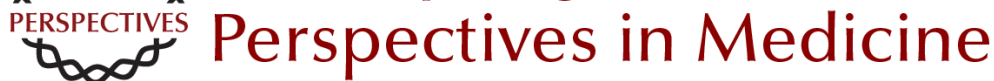

\section{Bat-Borne Influenza A Viruses: An Awakening}

Kevin Ciminski and Martin Schwemmle

Cold Spring Harb Perspect Med 2021; doi: 10.1101/cshperspect.a038612 originally published online December 23, 2019

\section{Subject Collection Influenza: The Cutting Edge}

\section{Emerging HxNy Influenza A Viruses William J. Liu, Yan Wu, Yuhai Bi, et al. \\ Equine Influenza Thomas M. Chambers \\ Human Influenza Epidemiology Sukhyun Ryu and Benjamin J. Cowling}

Host Cell Factors That Interact with Influenza Virus Ribonucleoproteins Ecco Staller and Wendy S. Barclay Induction and Evasion of Type-I Interferon Responses during Influenza A Virus Infection Raquel Muñoz-Moreno, Carles Martínez-Romero and Adolfo García-Sastre

Structure and Function of Influenza Polymerase Joanna M. Wandzik, Tomas Kouba and Stephen Cusack

H7N9 Influenza Virus in China Chengjun Li and Hualan Chen

\section{H5 Influenza Viruses in Egypt} Rabeh El-Shesheny, Ahmed Kandeil, Ahmed Mostafa, et al.

\author{
Antivirals Targeting the Neuraminidase \\ Larisa Gubareva and Teena Mohan \\ Accessory Gene Products of Influenza A Virus \\ Rute M. Pinto, Samantha Lycett, Eleanor Gaunt, et \\ al. \\ Influenza Immunization in the Context of \\ Preexisting Immunity \\ Susanne L. Linderman, Ali H. Ellebedy, Carl Davis, \\ et al. \\ Hemagglutinin Structure and Activities \\ Steven J. Gamblin, Sébastien G. Vachieri, Xiaoli \\ Xiong, et al. \\ Live Attenuated Cold-Adapted Influenza Vaccines \\ Kanta Subbarao \\ Next-Generation Influenza Vaccines \\ Masaru Kanekiyo and Barney S. Graham
Selective Genome Packaging Mechanisms of Influenza A Viruses Takeshi Noda \\ Systems Biological Analysis of Immune Response \\ to Influenza Vaccination \\ Mario Cortese, Amy C. Sherman, Nadine G. \\ Rouphael, et al.
}

For additional articles in this collection, see http://perspectivesinmedicine.cshlp.org/cgi/collection/ 